\title{
Assinatura geoelétrica de uma área contaminada por hidrocarbonetos
}

\author{
Daniela Antunes Ferreira*, Vagner Roberto Elis e Andréa Teixeira Ustra, IAG-USP
}

Copyright 2019, SBGf - Sociedade Brasileira de Geofísica

This paper was prepared for presentation during the $16^{\text {th }}$ International Congress of the Brazilian Geophysical Society held in Rio de Janeiro, Brazil, 19-22 August 2019.

Contents of this paper were reviewed by the Technical Committee of the $16^{\text {th }}$ International Congress of the Brazilian Geophysical Society and do not necessarily represent any position of the SBGf, its officers or members. Electronic reproduction or storage of any part of this paper for commercial purposes without the written consent of the Brazilian Geophysical Society is prohibited.

\section{Abstract}

This work presents the results of a geoelectric investigation conducted in a former wood treatment plant contaminated with creosote oil, in São Paulo city, Brazil. The direct-current resistivity, induced polarization and capacitively coupled resistivity methods were used. The results suggest that high resistivity anomalies are caused by the presence of creosote in the soil and low resistivity anomalies are consequences of electrical properties changes of the contaminated environment, due to biodegradation. Both methods contributed to the characterization of the study area.

\section{Introdução}

A contaminação da subsuperfície por hidrocarbonetos é alvo de grande preocupação ambiental e consiste em uma das principais fontes de contaminação das águas subterrâneas no mundo. Tradicionalmente, a investigação geoambiental de áreas contaminadas por hidrocarbonetos é feita através de métodos invasivos, que incluem as sondagens e a perfuração de poços. No entanto, a execução de tais técnicas costuma ser demorada e dispendiosa. Para aperfeiçoar o modelo conceitual da área contaminada, é possível integrar os métodos tradicionais com os métodos geofísicos, que além de minimamente invasivos, apresentam tempo e custo de execução menores (FREITAS et al., 2015).

Há uma série de métodos geofísicos que podem ser empregados em estudos ambientais, porém os mais indicados para a detecção e mapeamento de plumas de contaminação são os métodos geoelétricos (IPT, 2014). Isso porque em derrames de hidrocarbonetos, espera-se um contraste de resistividade entre a zona impactada e o meio geológico. Ainda, o processo de biodegradação altera as propriedades dos solos e rochas, modificando a assinatura geofísica do ambiente contaminado por hidrocarbonetos (ATEKWANA e ATEKWANA, 2010).

A Figura 1 apresenta o modelo conceitual da pluma de contaminação formada por um derrame de hidrocarbonetos. Em subsuperfície, os contaminantes derivados de hidrocarbonetos são dispostos como um líquido orgânico conhecido como NAPL (fase líquida não aquosa), que pode ser classificado em LNAPL (fase líquida não aquosa mais leve que a água) ou DNAPL (fase líquida não aquosa mais densa que a água). O transporte de NAPLs no meio se dá através das fases dissolvida, vapor, adsorvida, livre e residual (LAGO, 2009).

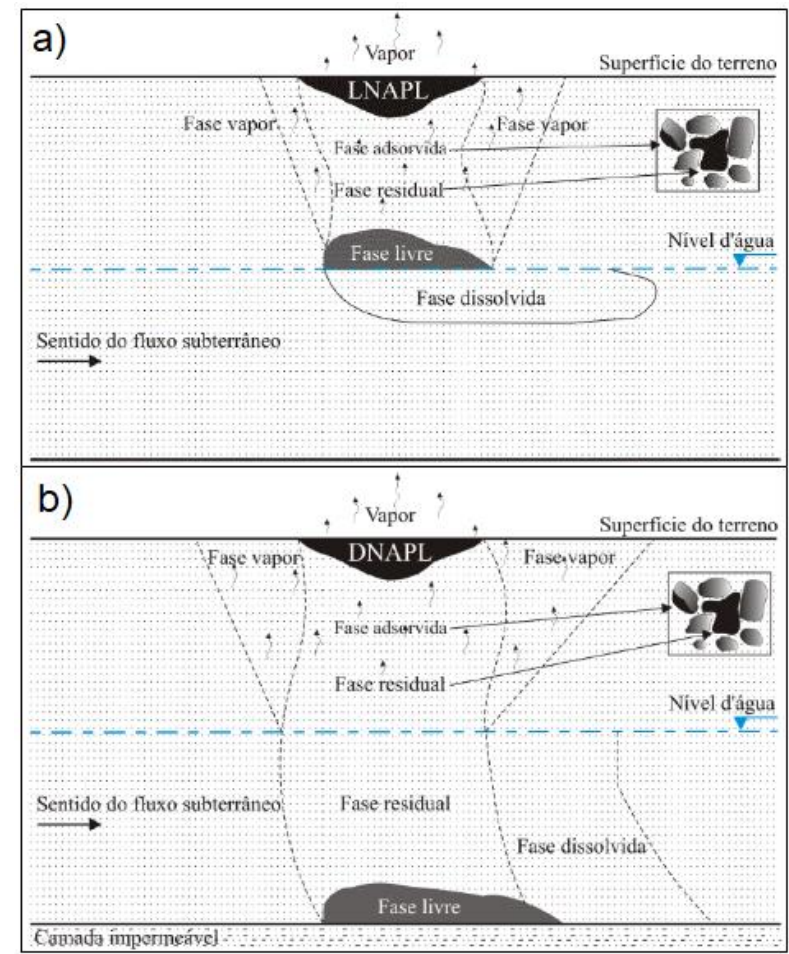

Figura 1. Desenho esquemático do comportamento dos NAPLs em subsuperfície. a) fase líquida não aquosa leve (LNAPL). b) fase líquida não aquosa densa (DNAPL) (Lago, 2009).

Este trabalho apresenta os resultados da investigação geoelétrica conduzida em uma antiga usina de tratamento de madeiras contaminada por óleo de creosoto. Foram utilizados os métodos da eletrorresistividade (ER), da polarização induzida (IP) e da resistividade capacitiva $(\mathrm{RC})$. O método IP tem sido cada vez mais empregado em estudos ambientais e uma investigação realizada por Kress \& Teeple (2005) numa área contaminada em Houston, Texas, mostrou que é possível detectar o óleo de creosoto através dos métodos ER e RC.

\section{Área de estudo}

A área de estudo está localizada no bairro do Jaguaré, São Paulo - SP (Figura 2), em um terreno que abrigou entre 1974 e 1997 uma usina de tratamento de madeiras. 


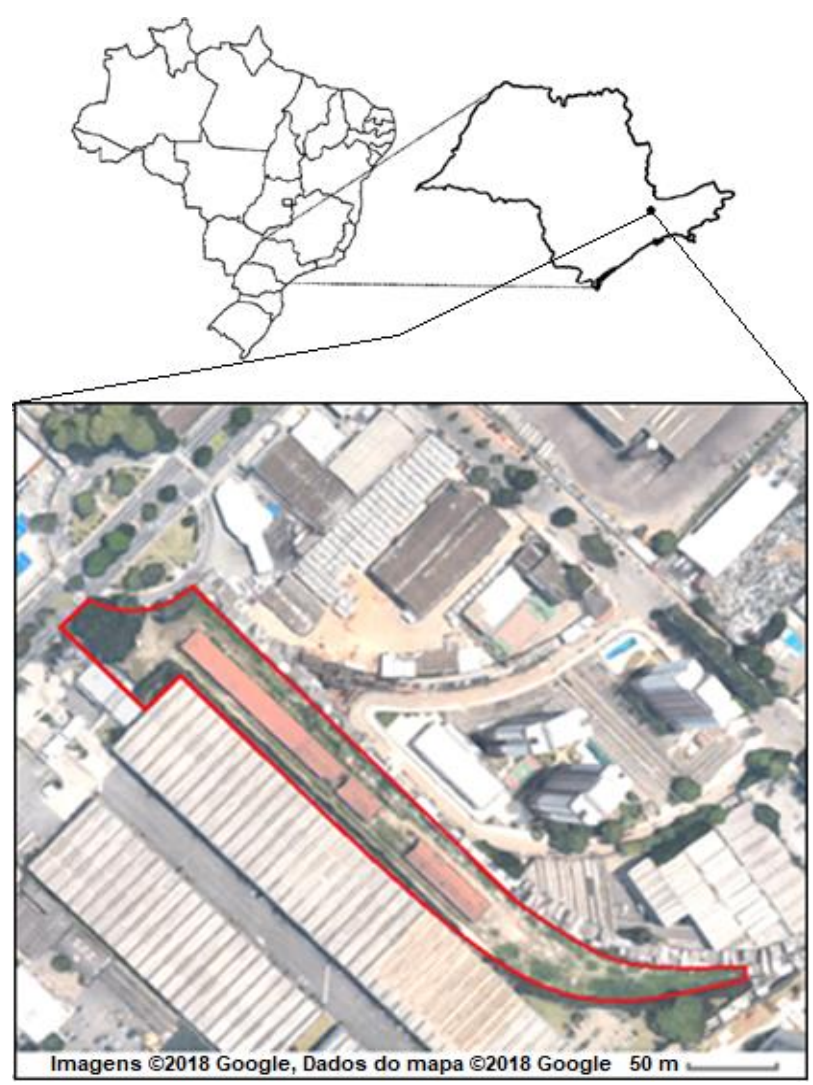

Figura 2. Localização da área de estudo (em vermelho) no bairro do Jaguaré, São Paulo - SP.

Durante o período de operação da usina, o vazamento de tanques aéreos, bem como o manuseio de produtos utilizados na preservação química da madeira, levou à contaminação do solo, confirmada em 2011 através de uma investigação do Instituto de Pesquisas Tecnológicas (IPT). Os principais contaminantes no local são compostos por hidrocarbonetos, principalmente o óleo de creosoto, e em menor extensão por produtos químicos inorgânicos como o pentaclorofenato de sódio e o arseniato de cobre cromatado (FREITAS et al., 2015).

O creosoto usado na preservação de madeiras é um óleo de cor escura e odor característico, derivado da destilação do alcatrão de hulha. O óleo de creosoto pode causar efeitos adversos à saúde humana, como irritação nos olhos, pele, aparelho respiratório e digestivo, através do contato, ingestão e inalação. Além disso, há evidências da sua carcinogenicidade em animais (IBAMA, 2015).

A geologia do local é caracterizada por depósitos aluvionares quaternários, sob os quais se encontram os depósitos terciários da Bacia de São Paulo. Em geral, uma camada de aterro consistindo de material argiloarenoso, com aproximadamente $2 \mathrm{~m}$ de espessura, sobrepõe as camadas de argila, areia e cascalho com granodecrescência ascendente, isto é, a dimensão do grão diminui em direção às camadas de topo. Em meio às camadas de areia, há lentes de argila (FREITAS et al.,
2015). A profundidade média do nível de água na área de estudo é de aproximadamente $4 \mathrm{~m}$.

Com base nos dados de investigação direta (não apresentados aqui), foram estabelecidas duas linhas de aquisição (Figura 3). A linha 1 é referente ao background (meio não contaminado), enquanto a linha 2 é referente ao meio contaminado.

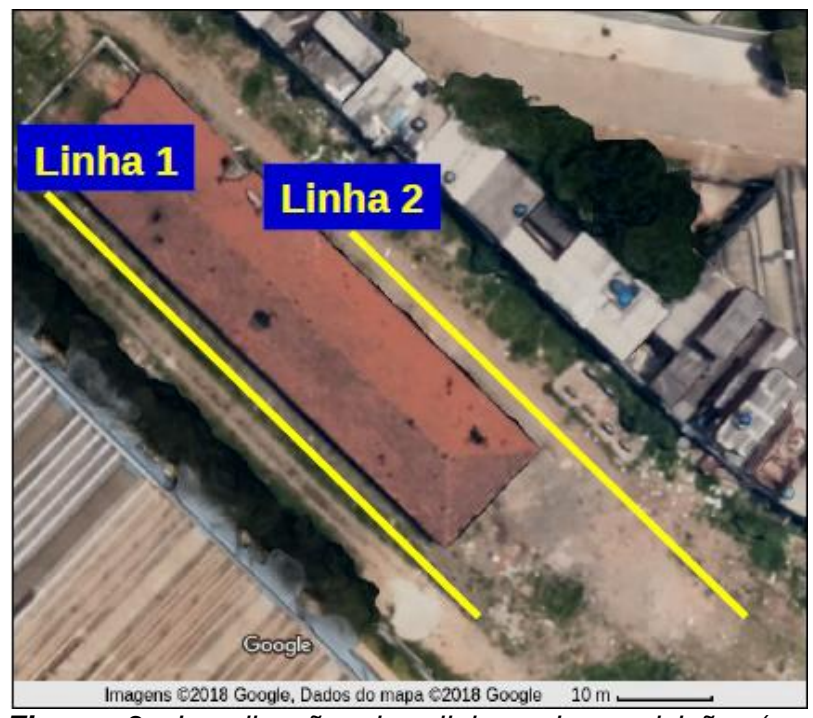

Figura 3. Localização das linhas de aquisição (em amarelo) na área de estudo.

\section{Métodos}

Para obter a assinatura geoelétrica da área de estudo, foram utilizados três métodos geofísicos: eletrorresistividade (ER), polarização induzida (IP) e resistividade capacitiva $(R C)$.

O método ER consiste em introduzir uma corrente elétrica gerada artificialmente no solo e medir as diferenças de potencial resultantes na superfície. Com isso, é possível obter informações sobre as propriedades elétricas da subsuperfície, como a resistividade real ou aparente (KEAREY et al., 2009). Sabe-se que os hidrocarbonetos apresentam grande contraste de resistividade em relação ao meio não contaminado. Em geral, vazamentos recentes apresentam anomalias resistivas, enquanto vazamentos antigos apresentam anomalias condutivas, devido ao processo de biodegradação (LAGO, 2009).

Quando a corrente elétrica aplicada é desligada, a voltagem entre os eletrodos de potencial sofre um grande decréscimo inicial, seguido por um decaimento gradual. Isso ocorre porque o meio armazena cargas elétricas, ou seja, se torna polarizado. Através desse fenômeno, chamado de polarização induzida, é possível obter a cargabilidade do terreno (KEAREY et al., 2009). O método IP é sensível às propriedades físicas do meio poroso e aos processos eletroquímicos que ocorrem nas interfaces mineral-fluido (NTARLAGIANNNIS et al., 2018). Vazamentos recentes geralmente apresentam anomalias de baixa cargabilidade, enquanto vazamentos 
antigos apresentam anomalias de alta cargabilidade (LAGO, 2009).

A aplicação dos métodos ER e IP se deu por meio da técnica do caminhamento elétrico, que investiga variações laterais de parâmetros físicos (nesse trabalho, variações da resistividade e da cargabilidade), a uma ou mais profundidades (BRAGA, 2016). Os dados foram coletados entre abril e maio de 2018 com o resistivímetro Syscal Pro (Iris Instruments), investigando 8 níveis teóricos de profundidade. Utilizou-se o arranjo dipolodipolo com espaçamento de $2.5 \mathrm{~m}$ metros entre os eletrodos.

O método ER apresenta limitações em terrenos pavimentados (como é o caso dessa área de estudo), pela necessidade de inserir eletrodos no terreno e estabelecer contato galvânico com o meio. O método RC se apresenta como uma alternativa viável nessas situações, pois a corrente (AC) é conduzida através do acoplamento capacitivo. As medidas são realizadas sob condições quasi-estáticas, nas quais o efeito indutivo pode ser desconsiderado. Nessas condições, os métodos ER e RC são essencialmente equivalentes (CAVENAGHI, 2017).

Um dos sistemas de aquisição que operam com o método RC é o OhmMapper (Geometrics), composto por um transmissor e um conjunto de receptores. Os receptores são conectados entre si através de cabos dipolares (que equivalem aos eletrodos no método ER) e o transmissor é ligado ao conjunto de receptores por meio de uma corda não condutiva, cujo comprimento pode variar. Quanto maior o comprimento da corda, maior a profundidade investigada. Todo o arranjo é rebocado por um operador ou por um veículo (CAVENAGHI, 2017).

Nesse trabalho, foi utilizada a técnica de aquisição contínua, na qual os dados são adquiridos em intervalos de tempo pré-definidos e a densidade de medidas realizadas depende da velocidade de locomoção do operador (CAVENAGHI, 2017). O intervalo de tempo adotado para a aquisição de dados foi de $500 \mathrm{~ms}$. A aquisição contínua com o OhmMapper foi realizada em setembro de 2018, com marcadores de posição a cada 5 $\mathrm{m}$ percorridos na linha, utilizando o arranjo dipolo-dipolo e três comprimentos de corda não condutiva: $2.5 \mathrm{~m}, 5 \mathrm{~m}$ e $10 \mathrm{~m}$.

Os dados obtidos foram baixados e processados de forma preliminar nos softwares Prosys II (Geomatrix) e MagMap (Geometrics). Em seguida, os modelos de resistividade e cargabilidade em subsuperfície foram desenvolvidos utilizando o software RES2DINV (Geotomo), que emprega uma rotina de inversão baseada no método dos mínimos quadrados com vínculo de suavização (GEOTOMO SOFTWARE, 2018).

\section{Resultados e discussão}

A Figura 4 apresenta a seção do modelo de resistividade gerado pelo processo de inversão dos dados da Linha 1, obtidos com o método ER. As cores quentes (vermelho, violeta) indicam alta resistividade, enquanto as cores frias (azul, verde) indicam baixa resistividade. Essa mesma escala de cores foi utilizada nas imagens 6, 7 e 9, para que os modelos obtidos pudessem ser comparados. Após 3 iterações, o erro RMS da inversão permaneceu alto $(21.3 \%)$. A faixa predominante de resistividades no modelo inverso está entre 50 e 150 ohm.m. Uma anomalia de baixa resistividade foi detectada entre 37.5 e $45 \mathrm{~m}$ do perfil, mas as análises químicas não detectaram contaminação no local. Suspeita-se que tal anomalia tenha sido causada pela má colocação de alguns eletrodos no solo, devido a pavimentação do terreno.

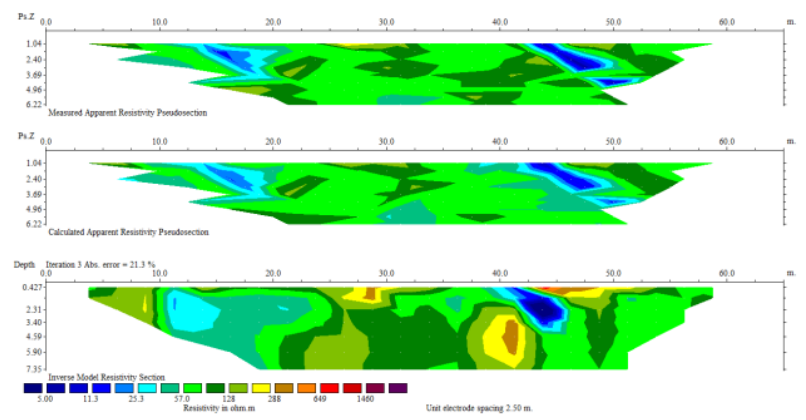

Figura 4. Pseudoseções de resistividade aparente medida e calculada e seção de resistividade do modelo inverso da Linha 1, obtidos com o método ER.

A Figura 5 apresenta a seção do modelo de cargabilidade gerado pelo processo de inversão dos dados da Linha 1, obtidos com o método IP. As cores quentes indicam alta cargabilidade, ao passo que as cores frias indicam baixa cargabilidade. Após 3 iterações, o erro RMS da inversão permaneceu baixo (6.5\%). No entanto, é possível notar o efeito do eletrodo mal colocado no solo entre 37.5 e $45 \mathrm{~m}$ do perfil. Com esse método, foi possível identificar o nível d'água em aproximadamente $4 \mathrm{~m}$ de profundidade.

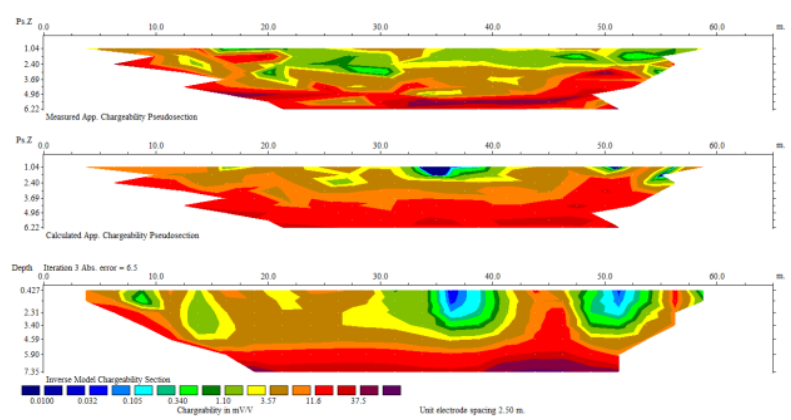

Figura 5. Pseudoseções de cargabilidade aparente medida e calculada e seção de cargabilidade do modelo inverso da Linha 1, obtidos com o método IP.

A Figura 6 apresenta a seção do modelo de resistividade gerado pelo processo de inversão dos dados da Linha 1, obtidos com o método RC. Após 4 iterações, o erro RMS da inversão permaneceu baixo (6.3\%). A faixa predominante de resistividades no modelo inverso também está entre 50 e 150 ohm.m e a profundidade investigada com o método $\mathrm{RC}(6.22 \mathrm{~m})$ é um pouco menor do que a profundidade investigada com o método ER $(7.35 \mathrm{~m})$. Nenhuma anomalia significativa foi 
detectada no perfil, o que era esperado para a linha de background.

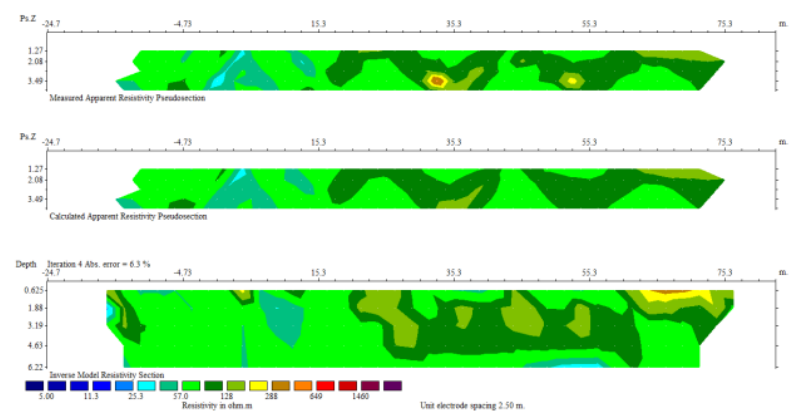

Figura 6. Pseudoseções de resistividade aparente medida e calculada e seção de resistividade do modelo inverso da Linha 1, obtidos com o método RC.

A Figura 7 apresenta a seção do modelo de resistividade gerado pelo processo de inversão dos dados da Linha 2, obtidos com o método ER. Após 4 iterações, o erro RMS da inversão permaneceu em $14.8 \%$. No centro do perfil (entre 30 e $45 \mathrm{~m}$ ), foi detectada uma anomalia condutiva com resistividade inferior a 25 ohm.m, acompanhada por uma anomalia resistiva, com resistividade acima de 288 ohm.m. Como se trata de um vazamento antigo, a anomalia resistiva indica a zona contaminada por hidrocarbonetos, enquanto a anomalia condutiva indica a zona onde os hidrocarbonetos já sofreram biodegradação. Também no centro do perfil, mas a uma profundidade maior que $4 \mathrm{~m}$, é possível observar outra anomalia resistiva, que pode estar relacionada à fase residual, com o creosoto retido nas lentes de argila.

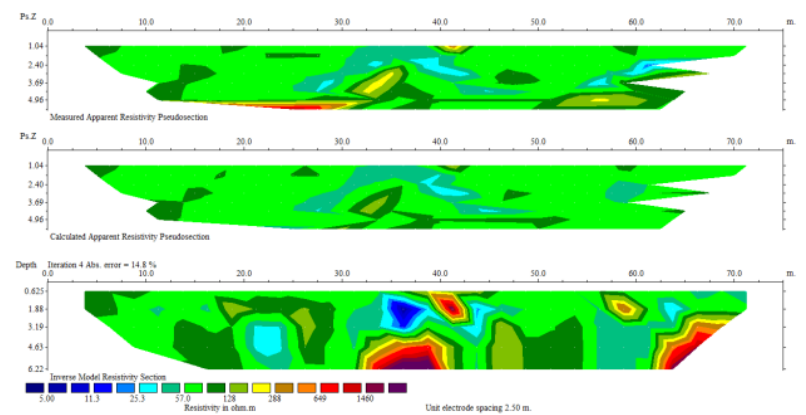

Figura 7. Pseudoseções de resistividade aparente medida e calculada e seção de resistividade do modelo inverso da Linha 2, obtidos com o método ER.

A Figura 8 apresenta a seção do modelo de cargabilidade gerado pelo processo de inversão dos dados da Linha 2 obtidos com o método IP. A escala de cores é a mesma que foi utilizada na Figura 5. Após 4 iterações, o erro RMS da inversão permaneceu baixo (5.6\%). Com esse método, o nível d'água foi identificado em aproximadamente $4 \mathrm{~m}$ de profundidade. No centro do perfil, é possível notar o aumento da cargabilidade devido a presença do creosoto.

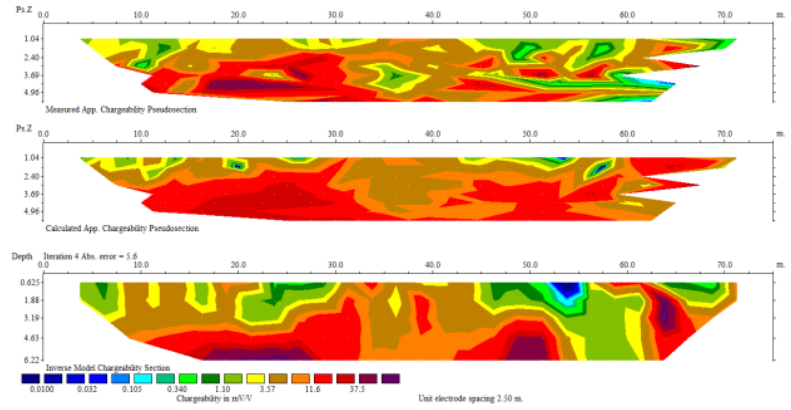

Figura 8. Pseudoseções de cargabilidade aparente medida e calculada e seção de cargabilidade do modelo inverso da Linha 2, obtidos com o método IP.

Por fim, a Figura 9 apresenta a seção do modelo de resistividade gerado pelo processo de inversão dos dados da Linha 2, obtidos com o método RC. Após 4 iterações, o erro RMS da inversão permaneceu em $11.9 \%$. Aproximadamente no centro do perfil, foi detectada uma anomalia condutiva com resistividade inferior a 25 ohm.m, acompanhada por uma anomalia resistiva, com resistividade acima de 288 ohm.m. Essa anomalia pode ser correlacionada àquela observada na Figura 7.

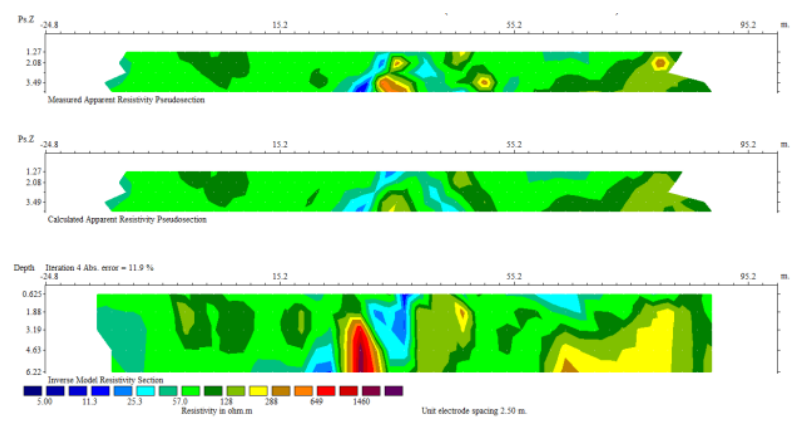

Figura 9. Pseudoseções de resistividade aparente medida e calculada e seção de resistividade do modelo inverso da Linha 2, obtidos com o método $R C$.

\section{Conclusões}

Os resultados obtidos nesse trabalho demonstram que os três métodos utilizados permitiram determinar 0 background geoelétrico da área de estudo e detectar a presença do contaminante no solo.

$\mathrm{Na}$ linha 1 (background), através dos métodos ER e RC, identificou-se a faixa predominante de resistividades no meio, que varia de 50 a 150 ohm.m. Com o método IP, foi possível identificar o nível d'água em aproximadamente 4 $\mathrm{m}$ de profundidade.

$\mathrm{Na}$ linha 3 (referente ao meio contaminado), os métodos ER e RC detectaram anomalias de alta resistividade, relacionadas à presença do óleo de creosoto, e anomalias de baixa resistividade, interpretadas como o resultado da exposição do contaminante ao processo de biodegradação. 
Apesar de investigar uma profundidade menor, o método $\mathrm{RC}$ apresentou vantagens em relação ao método ER. Por utilizar o acoplamento capacitivo, o método $R C$ não foi prejudicado pela pavimentação do terreno. Além disso, a aquisição de dados com esse método foi mais rápida. Por outro lado, o caminhamento elétrico permitiu a aplicação conjunta dos métodos ER e IP, o que contribuiu para a caracterização da área estudada.

Tendo em vista as suas vantagens operacionais e a sua eficiência em detectar as zonas impactadas pelo contaminante, a aplicação do método RC pode ser considerada no monitoramento ambiental da área de estudo.

\section{Agradecimentos}

Ao Alexandre Barbosa, pesquisador do IPT, pelo acesso à área de estudo. Aos técnicos do IAG-USP, Ernande Costa Santos e Marcelo César Stangari, pela contribuição durante os trabalhos de campo.

\section{Referências}

ATEKWANA, E.A. \& ATEKWANA, E.A., 2010. Geophysical signatures of microbial activity at hydrocarbon contaminated sites: A review. Surveys in Geophysics, 31: 247-283. DOI:10.1007/s10712-0099089-8.

BRAGA, A. C. O., 2016. Geofísica aplicada: Métodos Geoelétricos em Geofísica. São Paulo: Editora Oficina de Textos.

CAVENAGHI, V. L. S., 2017. Caracterização geoelétrica de alvos rasos no Sítio Controlado de Geofísica Rasa-II IAG/USP através do uso de resistividade capacitiva, Dissertação de Mestrado, Instituto de Astronomia, Geofísica e Ciências Atmosféricas, Universidade de São Paulo, São Paulo - SP, Brasil.

FREITAS, L. G., GANDOLFO, O. C. B., GALLI, V. L., BLANCO, R. G., BARBOSA, A. M. \& LEITE, D. C., 2015, Integração de Métodos Geofísicos com a sonda MIP para caracterização estratigráfica de uma área contaminada, $15^{\circ}$ Congresso Brasileiro de Geologia e Engenharia Ambiental.

GEOTOMO SOFTWARE. RES2DINV, 2018, version 4.08, Rapid 2-D resistivity \& IP inversion using the leastsquare method - Geoelectrical Imaging 2-D \& 3-D, Geotomo Software, Penang, Malaysia.

IBAMA - Instituto brasileiro de Meio Ambiente e dos Recursos Naturais Renováveis, 2015. Ficha de informações de segurança de produtos químicos - Óleo de preservação de madeira. Disponível em: <https://www.ibama.gov.br/component/phocadownload/fil e/2887-oleo-creosoto>. Acesso em: 18 de março de 2019.

IPT - Instituto de Pesquisas Tecnológicas do Estado de São Paulo, 2014. Guia de elaboração de planos de intervenção para o gerenciamento de áreas contaminadas. Disponível em: <https://www.ipt.br/download.php?filename=1159-

Guia_Gerenciamento_de_Areas_Contaminadas_1a _edicao_revisada.pdf>. Acesso em: 18 de março de 2019.

KEAREY, P., BROOKS, M., \& HILL, I., 2009. Geofísica de Exploração. São Paulo: Editora Oficina de Textos.

KRESS, W. H., \& TEEPLE, A. P., 2005. Two-Dimensional Resistivity Investigation of the North Cavalcade Street Site, Houston, Texas, August 2003: U.S. Geological Survey Scientific Investigations Report 2005-5205, 32 p.

LAGO, A. L., 2004. Aplicação integrada de métodos geofísicos na área de disposição de resíduos sólidos urbanos em Bauru - SP. Dissertação de Mestrado. Instituto de Astronomia, Geofísica e Ciências Atmosféricas, Universidade de São Paulo, São Paulo SP, Brasil, 110p.

NTARLAGIANNNIS, D., USTRA, A., KESSOURI, P., FLORES-OROZCO, A., 2018. The Untapped Potential of the Induced Polarization Method: Characterizing and Monitoring Hydrocarbon Contamination in Soils. Fast TIMES, 23(4): 64-73. 